Supporting Information for

\title{
Vertical Diffusion of Ions within Single Particles during Electrochemical Charging
}

Tinglian Yuan, Wei Wei, Wenxuan Jiang and Wei Wang*

State Key Laboratory of Analytical Chemistry for Life Science, Chemistry and Biomedicine Innovation Center, School of Chemistry and Chemical Engineering, Nanjing University, Nanjing 210023, China.

* Corresponding email: wei.wang@ nju.edu.cn

\section{Table of Contents}

S1. Materials

S2. Synthesis and characterization of PB colloidal

S3. da-TIRM apparatus

S4. Imaging processing method

S5. Detection sensitivity of TIRM

S6. Evaluation of the effect of angle and polarization on TIRM imaging

S7. Sample preparation for electrochemical experiments

S8. Cyclic voltammetry of ensemble PB particles

$\mathrm{S} 9$. Evaluation of the effect of $\mathrm{Pt}$ film on determining ionic diffusion direction

S10. Simulation of the electrochromic process of a PB particle

S11. Simulation of the longitudinal motions of a diffusing PS particle

S12. TIRM vs SPRM

S13. Descriptions for Movie S1 and Movie S2

References 


\section{S1. Materials}

Potassium nitrate $\left(\mathrm{KNO}_{3}\right)$ and potassium hexacyanoferrate ( II ) trihydrate $\left(\mathrm{K}_{4}[\mathrm{Fe}(\mathrm{CN})]_{6}\right)$ were purchased from Nanjing Chemical Reagent Co., Ltd, China. Hydrochloric acid ( $\mathrm{HCl})$ aqueous solution was purchased from Shanghai Hushi Chemical Reagent Co., Ltd, China. Suspension of 500-nm polystyrene (PS) nanospheres was purchased from Shanghai Huge Biotechnology Co., Ltd, China. In imaging the Brownian motions of PS nanospheres, 0.01 $\mathrm{M} \mathrm{KNO}_{3}$ aqueous solution was prepared to increase the ionic strength.

\section{S2. Synthesis and characterization of PB colloidal}

The PB colloidal used in electrochemical experiments were synthesized referring to a reported literature ${ }^{1}$. Briefly, $100 \mathrm{~mL}$ of mixed reaction solution containing $1 \mathrm{mM}$ $\mathrm{K}_{4}[\mathrm{Fe}(\mathrm{CN})]_{6}$ and $0.1 \mathrm{M} \mathrm{HCl}$ was put in water bath at $36{ }^{\circ} \mathrm{C}$ under ultrasonic condition for $90 \mathrm{~min}$. After cooling the sol to room temperature, the blue product was then washed repeatedly with deionized water $(18.2 \mathrm{M} \Omega \cdot \mathrm{cm})$ and centrifuged three times to remove the supernatant liquor. Finally, the as-synthesized colloidal was dried in a vacuum oven at $30{ }^{\circ} \mathrm{C}$ for $12 \mathrm{hr}$. Morphological characterization and UV-Vis extinction spectrum of PB particles are provided in Figure S1.

a

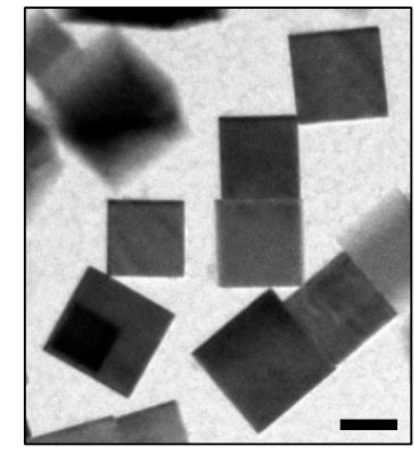

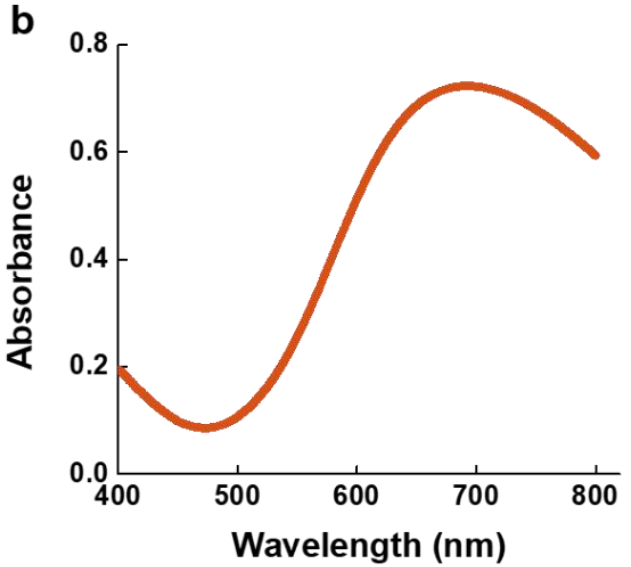

Figure S1. Morphological characterization and UV-Vis spectrum of PB colloidal. (a) TEM photo of PB particles. Scale bar: $200 \mathrm{~nm}$. (b) UV-Vis spectrum of PB colloidal with a wide absorption band around $690 \mathrm{~nm}$.

\section{S3. da-TIRM apparatus}

As Figure 3a shows, the present da-TIRM was built on a commercial inverted and dualdeck microscope (Nikon, Ti2-E) integrating with two TIRF illuminators and an oilimmersion objective (Nikon, 60×, N.A.=1.49). Two superluminescent diode (SLD) light sources (Qphotonics L.L.C, QSDM-680-2) with the same wavelength of $680 \mathrm{~nm}$ were mounted in the TIRF illuminators. One of the beams formed $p$-polarized light after passing 
through a polarizer (Edmund Optics, \#54-926) at an incident angle $\theta_{2}$ (i.e., $p$-, $\theta_{2}$ ) while another beam formed $s$-polarized light that was manipulated by a polarizer and a half-wave plate (Thorlabs, WPH10E-670) at $\theta_{1}$ (i.e., $s-, \theta_{1}$ ). Each light beam was reflected by a beamsplitter (Thorlabs, BSW26R) to enter into the objective, then collimated and simultaneously illuminated the samples. Subsequently, the reflected beams were separated into independent two again by an image splitter device (Cairn, Optosplit II ) inside which a polarizing beamsplitter (PBS) cube was integrated, to further hit a charge-coupled device (CCD) camera (Allied Vision Technology, Stingray F-046) after passing through a redpass filter (Semrock, BLP01-594R-25). Eventually, a wide-field da-TIRM image was generated in the same field of view at the same time. The power densities of the incident beams reaching the sample plane were measured as $0.2 \mathrm{~W} / \mathrm{cm}^{2}$.

As for the angle adjustment, $\theta_{1}$ was digitally controlled by Nikon software (NIS-Ar) with an accuracy of $0.1^{\circ}$ whereas $\theta_{2}$ was manually tuned. Hence, an accompanying pair of evanescent waves with two distinct angle-dependent illumination depths $\left(d_{1}\right.$ and $\left.d_{2}\right)$ was produced. In the present work, the range of the penetration depth was able to be determined from $100 \mathrm{~nm}$ to $1 \mu \mathrm{m}$ (Figure S2).

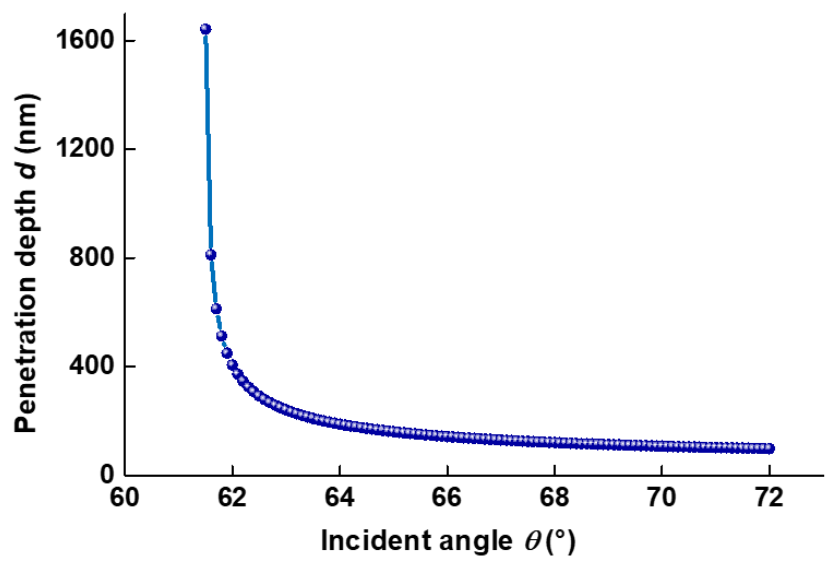

Figure S2. Curve of penetration depth $v s$ incident angle. The penetration depth refers to glass-water interface with refractive index $n_{\text {glass }}=1.515$ and $n_{\text {water }}=1.331$. The parameters used in the present work are listed in Table 1.

\section{S4. Imaging processing method}

A background subtraction method was adopted to calculate the TIRM intensity value of a particle. Figure S3a shows da-TIRM image of a PB particle on Pt-coated ITO cover slip during electrochemical cycling. The regions of interest (ROI) covering mixed signals from both particle and background (ROI 1 and 3), and background (ROI 2 and 4) are marked by rectangle frameworks with a dimension of $5 \times 20$ pixels $\left(1.2 \times 4.9 \mu \mathrm{m}^{2}\right)$. The raw intensity curves belonging to particle plus background (blue) and background (black) are extracted, as shown in Figure S3b and S3c. Note that the optical variations of the substrate come from the changes in electron density of Pt layer, since the dielectric constant of metal is dependent on the electron density. The optical responses (red) that the PB particle solely 
contributed to can be resolved by subtracting the values in the black curve by that of the blue curve. Finally, da-TIRM intensity curves are obtained by plotting the intensity curves solely from particle against time (Figure S3d).

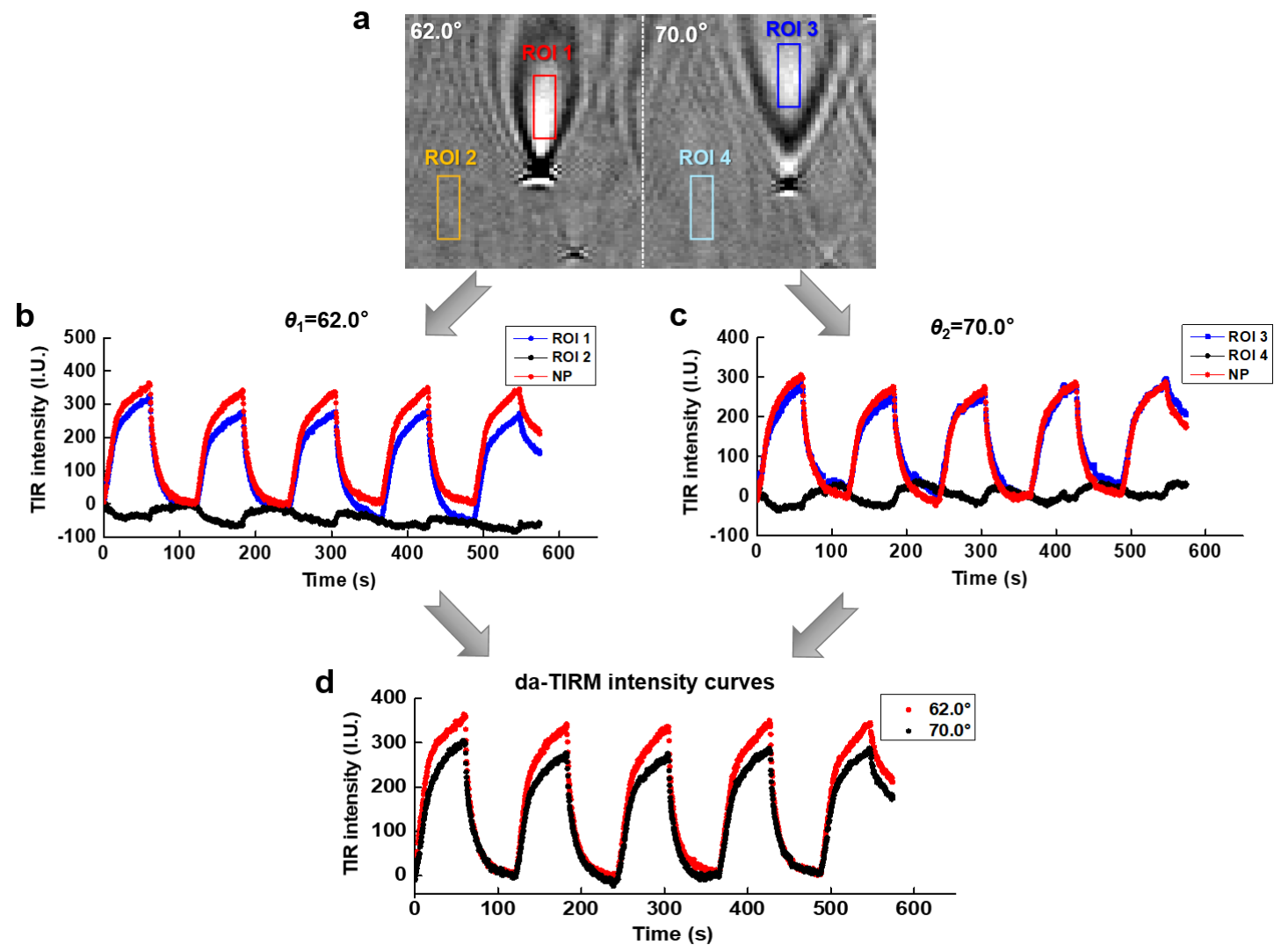

Figure S3. Image processing method. (a) da-TIRM image of a PB particle at $\boldsymbol{\theta}_{\mathbf{1}}=62.0^{\circ}$ (left panel) and $\boldsymbol{\theta}_{\mathbf{2}}=70.0^{\circ}$ (right panel). In the TIRM image at $62.0^{\circ}$, regions of interest (ROI) numbered with 1 and 2 represent the signal area in presence of particle (particle + background) and in absence of particle (background only), respectively. ROI 3 and 4 in the TIRM image at $70.0^{\circ}$ are done in the same manner. Two raw intensity curves calculated from ROI 1 (blue) and ROI 2 (black), plus the intensity curve of particle (red) by subtracting the black one from the blue one are presented in (b) $62.0^{\circ}$ and (c) $70.0^{\circ}$. (d) daTIRM intensity curves obtained from (b) and (c).

\section{S5. Detection sensitivity of TIRM}

In order to differentiate the kinetic differences between da-TIRM intensity curves, we measured different particles with various sizes below $1 \mu \mathrm{m}$ (comparable with the maximal penetration depth). Figure S4a presents SEM images of three PB particles ranging from $100 \mathrm{~nm}$ to $400 \mathrm{~nm}$, and their corresponding TIRM images. Figure S4b shows the intensity profile along the cross section drawn with a yellow line in particle \#3 TIRM image (140 $\mathrm{nm} \times 129 \mathrm{~nm}$ ), with a maximum peak value of -935 I.U. and a SNR of 26. 

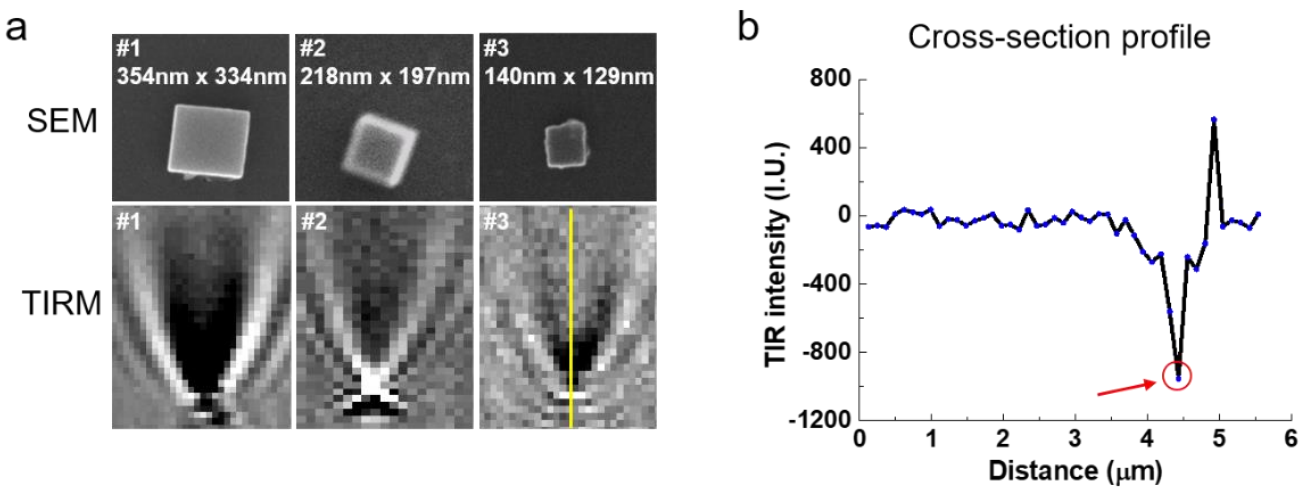

Figure S4. (a) SEM and TIRM images of three PB particles. (b) Optical intensity profile of particle \#3.

\section{S6. Evaluation of the effect of angle and polarization on TIRM imaging}

Some reshaping occurred between da-TIRM images because of incident angle rather than polarization state. To clarify this, we provided two pairs of da-TIRM images of 500$\mathrm{nm}$ PS spheres recorded at different angles $\left(61.6^{\circ}\right.$ or $\left.70.0^{\circ}\right)$ and with orthogonal polarization illumination states in Figure S5. At the same angle (left column vs right column), all the bright and dark spots are overlapped, illustrating that the shift is polarization-independent. Apart from that, in the theory of evanescent field, the penetration depth for $p$ and $s$ is identical ${ }^{2,3}$ so that the polarization state would not affect the measuring results. But for these TIRM patterns captured at different angles (top panel vs bottom panel), dislocation occurs in the shapes.
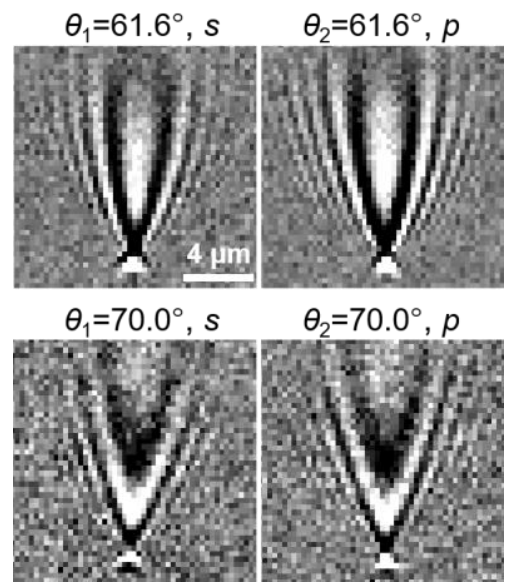

Figure S5. da-TIRM images of 500-nm PS particles (top panel vs bottom panel) at different angles and (left column $v s$ right column) with different polarizations. 


\section{S7. Sample preparation for electrochemical experiments}

The electrochemical experiments of single PB particles were performed with an electrochemical workstation (Wuhan Corrtest Instruments Co., CS310). When conducting experiments, we synchronized the voltage, image sequence and time by a data acquisition (DAQ) device (National Instrument, USB-6251). A three-electrode system was adopted, including ITO glass (SPI Supplies, \#1 $22 \mathrm{~mm} \times 22 \mathrm{~mm} \mathrm{15-30} \mathrm{Ohms)} \mathrm{served} \mathrm{as} \mathrm{working}$ electrode, Pt coil as counter electrode and Pt wire as quasi-reference electrode. ITO glass was soaked in Alconox detergent, iso-propanol, acetone and water successively, meanwhile purged under ultrasonic condition for $10 \mathrm{~min}$ for each cleaning solution. In prior to experiments, PB particles were immobilized on ITO surface via an electrostatic adsorption method. In order to obtain robust electrochemical responses from individual PB particles, we sprayed a thin layer $(\sim 5 \mathrm{~nm})$ of platinum to increase electrical contacts with the conductive substrate according to our recent reported study. ${ }^{4}$ A polydimethylsiloxane (PDMS) cell (Sarstedt, flexiPERM micro12) was then mounted on the ITO as an electrochemical cell, into which $200 \mu \mathrm{L}$ of $1 \mathrm{M} \mathrm{KNO}_{3}$ solution was added as electrolyte solution. Before performing step experiments, a potential of $0 \mathrm{~V}$ was held for $100 \mathrm{~s}$ to oxidize those partially reduced particles. Figure S6 shows the schematic diagram of daTIRM detection for the experimental experiments.

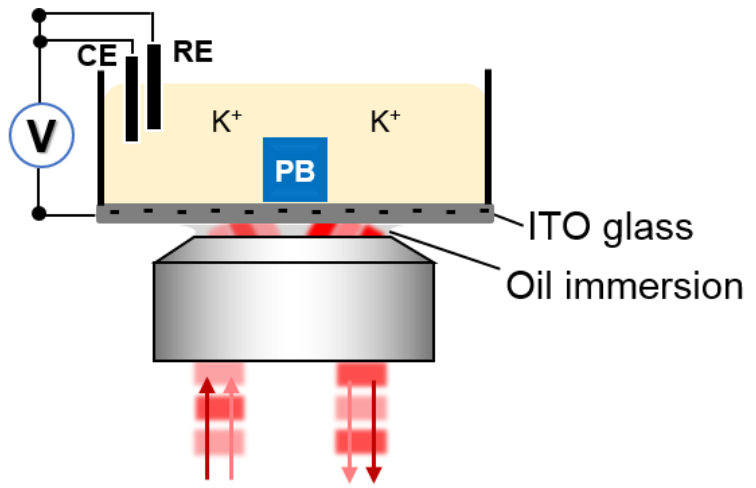

Figure S6. Experimental schematic diagram of imaging single PB particle electrochemistry.

\section{S8. Cyclic voltammetry of ensemble PB particles}

Before step experiments, the cyclic voltammetry of ensemble PB particles is measured (Figure S7) to ensure the oxidation and reduction potential. 


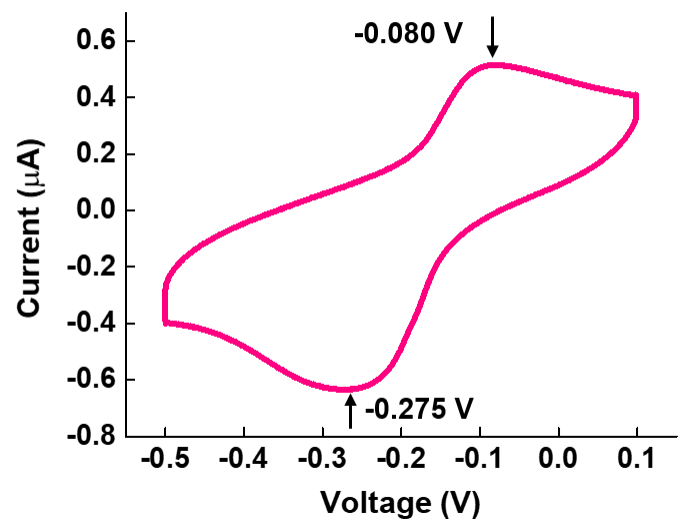

Figure S7. Cyclic voltammetry (CV) diagram of ensemble PB particles. CV of quantitative PB particles immobilized on ITO in $1 \mathrm{M} \mathrm{KNO}_{3}$, scan rate: $20 \mathrm{mV} / \mathrm{s}$. The apparent oxidation peak and reduction peak are located at $-0.080 \mathrm{~V}$ and $-0.275 \mathrm{~V}$, respectively.

\section{S9. Evaluation of the effect of $\mathrm{Pt}$ film on determining ionic diffusion direction}

In our previous studies (J. Am. Chem. Soc., 2020, 142, 14307), ${ }^{4}$ a layer of Pt film with thickness of $5 \mathrm{~nm}$ was used to strengthen the electrical contact between PB particles and electrode surface. We have demonstrated that the Pt-film exhibited a porous structure and did not block the ionic access at the side and top surfaces. In order to investigate the influence of Pt-film on the conclusions in the present study, we conducted additional experiments in the absence of Pt-film. Some of the particles exhibited satisfactory reactivity and cyclability even without the Pt-film. As shown in Figure S8 below, it was found that the outside-in model remains valid in the absence of Pt-film, though vertical case occurred occasionally (Figure S8h). The fundamental reason causing this result was attributed to the fast electron hopping along the particle shells.

a
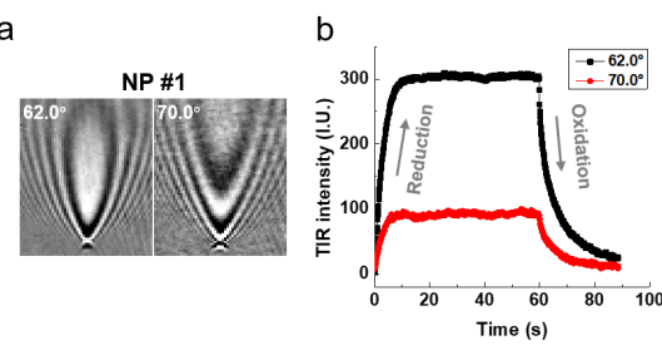

e

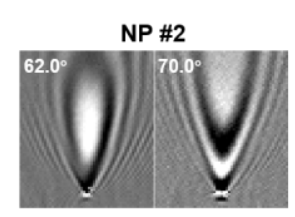

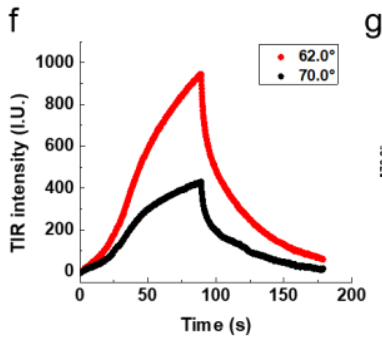
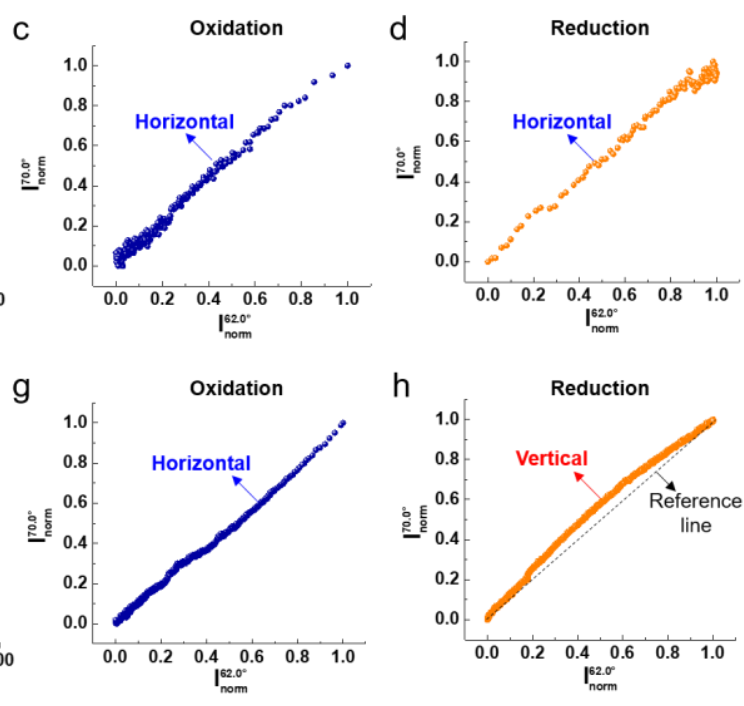
Figure S8. da-TIRM images, the corresponding intensity curves and $\boldsymbol{I}_{\boldsymbol{\theta} \mathbf{1}}-\boldsymbol{I}_{\boldsymbol{\theta} \mathbf{2}}$ plots of (a) (b) (c) (d) PB particle \#1 and (e) (f) (g) (h) \#2 immobilized on an ITO cover slip.

\section{S10. Simulation of the electrochromic process of a PB particle}

The vertical and horizontal ion-diffusion models (see Figure $2 \mathrm{a}$ and $2 \mathrm{~b}$ ) were simulated with MATLAB. In principle, the concentration distribution of PB component in the interior of the particle can be solved in accordance with Cottrell equation (Eq. 1 in the main text). By calculating the integral of the concentration gradient-relied intensity along the longitude and the weight in evanescent field, the intensity of a particle can be obtained. The simulation video is provided in Movie S1.

\section{S11. Simulation of the longitudinal motions of a diffusing PS particle}

The falling process of a 500-nm PS particle was simulated to further predict the heightrelated intensity changes under dual-angle detection condition $\left(\theta_{1}=62.0^{\circ}\right.$ and $\left.\theta_{2}=70.0^{\circ}\right)$ with MATLAB. During PS particle's falling process, its intensity value can be solved by calculating the integral of the volume-relied intensity and the weight in evanescent field. Figure S9 shows four groups of da-TIRM intensity pair.

a

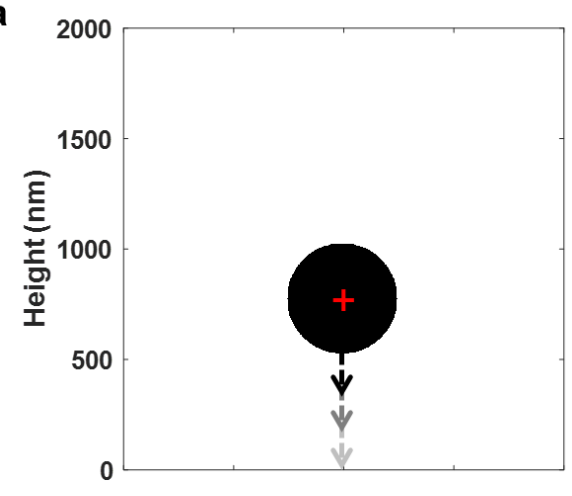

b

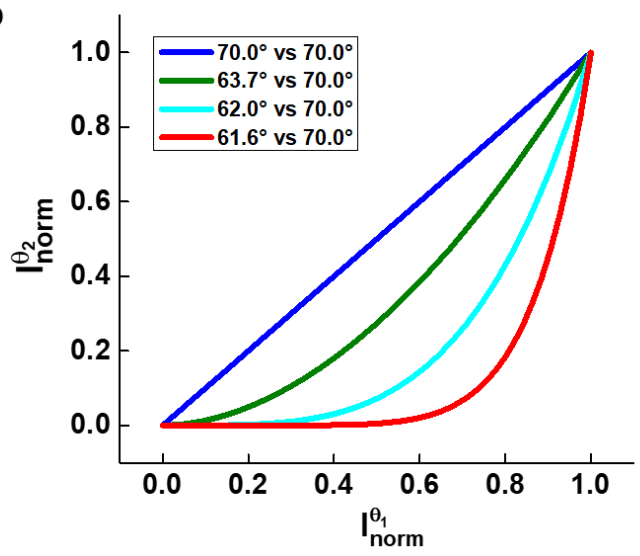

Figure S9. Simulated $\boldsymbol{I}_{\boldsymbol{\theta 1}}-\boldsymbol{I}_{\boldsymbol{\theta} \mathbf{2}}$ curves of a diffusing PS particle. $\boldsymbol{\theta}_{\mathbf{2}}$ was fixed at $70.0^{\circ}$ while $\boldsymbol{\theta}_{1}$ was $70.0^{\circ}, 63.7^{\circ}, 62.0^{\circ}$ and $61.6^{\circ}$, respectively. The relevant parameters are listed in Table 1.

\section{S12. TIRM vs SPRM}

We clarify the following three major differences between TIRM and SPRM on substrate, polarization and penetration depth, respectively. First, from the aspect of imaging principle, TIRM uses glass cover slip (in the absence of gold thin film, Figure S10a) as substrate and 
it is based on evanescent wave produced at the glass-medium interface to interact with particle, whereas SPRM employs gold-coated cover slip (Figure S10d) and relies on surface plasmon wave (SPW) generated at the gold-medium interface. Second, while SPRM only works under $p$-polarization illumination (S10a), TIRM is applicable for both p- and s-polarization with comparable sensitivities (S10d). In order to demonstrate this point, we scanned the incident angle from $62^{\circ}$ to $75^{\circ}$ to obtain the intensity curves of background (S10b, e) and a single particle (S10c, f), respectively. Third, as pointed out by this reviewer, the penetration depth of evanescent wave under TIR mode can be clearly described as an algebraic expression of incident angle. In contrast, such dependence of the penetration depth of surface plasmon wave on the incident angle is significantly complicated.

Table S1. Differences between TIRM and SPRM.

\begin{tabular}{c|cc}
\hline & TIRM & SPRM \\
\hline Substrate & cover slip (glass) & Gold-coated cover slip (glass) \\
Polarization & $p(\sqrt{ }), \mathrm{s}(\sqrt{ })$ & $p(\sqrt{ }), \mathrm{s}(\times)$ \\
$\begin{array}{c}\text { Penetration } \\
\text { depth }\end{array}$ & $\begin{array}{c}\text { Simple algebraic expression: } \\
d=\frac{\lambda}{4 \pi}\left(n_{1}^{2} \sin ^{2} \theta-n_{2}^{2}\right)^{-\frac{1}{2}}\end{array}$ & $\begin{array}{c}\text { Narrow depth range near dip angle; } \\
\text { Complicated solution }\end{array}$ \\
\hline
\end{tabular}

a
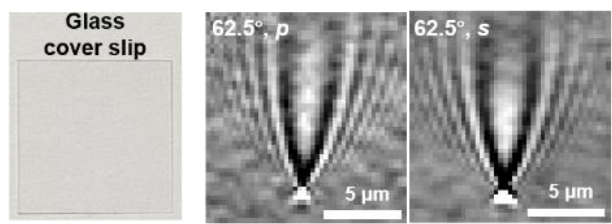

b
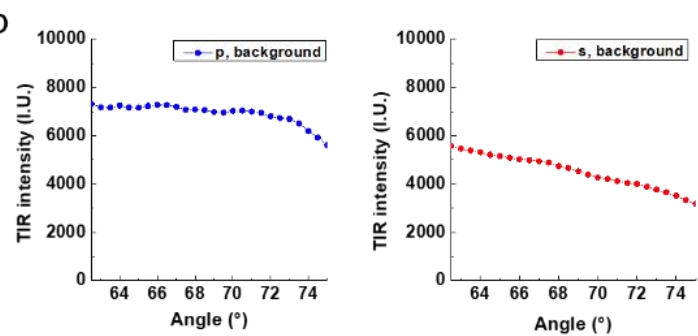

C
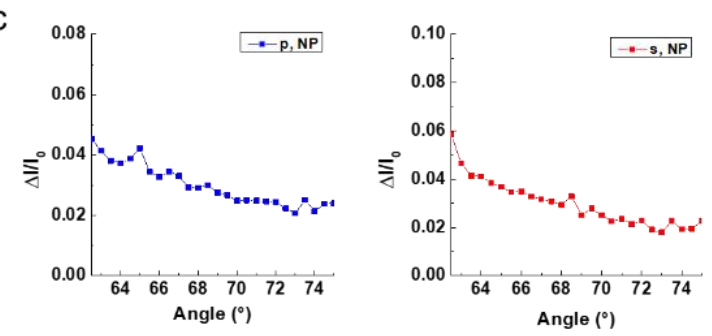

d
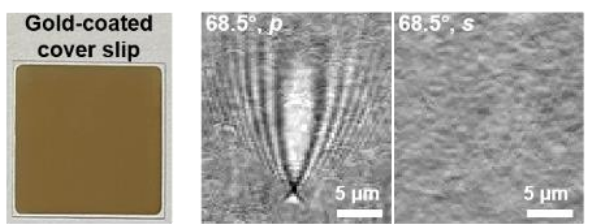

e
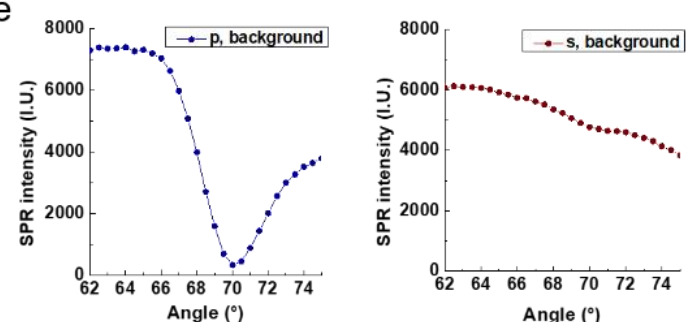

f
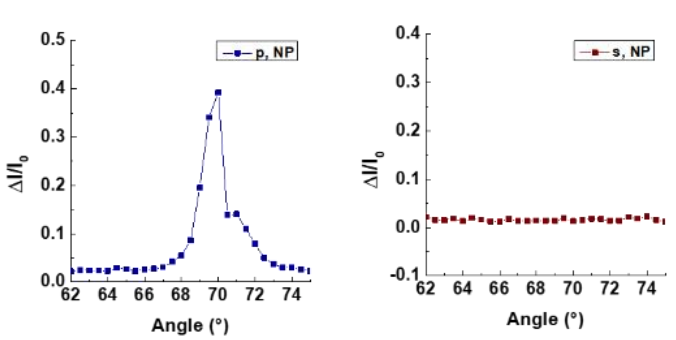
Figure S10. (a) Photograph of glass cover slip (in the absence of gold film) used for TIRM imaging, and TIRM images of a single particle under $p$ - and $s$-light illumination. TIR intensities $\mathrm{I}_{0}$ of background (b) and a particle (c) as a function of incident angle, respectively. (d) Photograph of gold-coated cover slip (thickness of gold film is $50 \mathrm{~nm}$ ) used for SPRM imaging, and SPRM images of a single particle under $p$ - and $s$-light illumination. SPR intensities $\mathrm{I}_{0}$ of background (e) and a particle (f) as a function of incident angle, respectively.

\section{S13. Descriptions for Movie S1 and Movie S2}

Movie S1 (separate file). Simulation of vertical and horizontal ion-diffusion models within a PB particle with MATLAB. $\theta_{1}$ and $\theta_{2}$ are to $62.0^{\circ}$ and $70.0^{\circ}$, respectively. The diffusion coefficient $D$ of $\mathrm{K}^{+}$was $1 \times 10^{-15} \mathrm{~m}^{2} / \mathrm{s}$. The simulated results are shown in Figure 2 .

Movie S2 (separate file). Monitoring the near-wall Brownian motions of a PS nanosphere by da-TIRM. The images and intensity curves are shown in Figure $3 \mathrm{~b}$ and 3c. Scale bar: 5 $\mu \mathrm{m}$.

\section{References}

(1) Wu, X. L.; Cao, M. H.; Hu, C. W.; He, X. Y., Sonochemical Synthesis of Prussian Blue Nanocubes from a Single-Source Precursor. Cryst. Growth Des. 2006, 6 (1), 26-28.

(2) Axelrod, D.; Burghardt, T. P.; Thompson, N. L., Total Internal Reflection Fluorescence. Annu. Rev. Biophys. Bioeng. 1984, 13, 247-68.

(3) Axelrod, D., Evanescent Excitation and Emission. In Fluorescence Microscopy; Cornea, A., Conn, P. M., Eds. Elsevier: Boston, 2014; pp 1-14.

(4) Wei, W.; Yuan, T.; Jiang, W.; Gao, J.; Chen, H.-Y.; Wang, W., Accessing the Electrochemical Activity of Single Nanoparticles by Eliminating the Heterogeneous Electrical Contacts. J. Am. Chem. Soc. 2020, 142 (33), 14307-14313. 